\title{
CALAGEM E SILICATAGEM EM SOLO INCUBADO COM DIFERENTES UMIDADES
}

\author{
Juliano Carlos Calonego, Vitor Savoia Mora, Carlos Henrique Santos, Leandro de Oliveira
}

Universidade do Oeste Paulista - UNOESTE

\section{RESUMO}

O calcário é o corretivo da acidez mais utilizado nos solos agrícolas brasileiros, porém benefícios importantes podem ser obtidos com o uso de silicatos de cálcio e magnésio. Objetivou-se comparar o efeito neutralizante da acidez do solo por meio de calagem e silicatagem, com incubação dos corretivos em condições de solo com diferentes umidades. O experimento foi conduzido no Laboratório de Análise Química de Solos, na Universidade do Oeste Paulista-UNOESTE, utilizando solo coletado na camada de 0 a $20 \mathrm{~cm}$ de um Argissolo Vermelho-Amarelado distrófico. Utilizou-se o delineamento experimental inteiramente casualizado, em esquema fatorial $2 \times 2 \times 5$, sendo dois corretivos (calcário dolomítico e silicato de $\mathrm{Ca}$ e $\mathrm{Mg}$ ), duas umidades do solo (80 e $20 \%$ da umidade de saturação) e cinco épocas de avaliação (60, 90, 120, 150 e 180 dias após incubação) dos atributos químicos do solo. Os resultados foram comparados por meio de análise de variância e as variáveis umidades do solo e corretivos foram submetidas ao teste de Tukey $(p<0,05)$. Já o efeito da época de amostragem foi analisado por meio de análise de regressão. $O$ silicato apresenta maior capacidade neutralizante da acidez do solo que 0 calcário, e com isso sua dosagem deve ser mais bem estudada, pois a utilização do cálculo para determinar a necessidade de calagem pode superestimar a necessidade de silicatagem. A reatividade do calcário no solo não é influenciada quando o solo está com umidade entre 20 e $80 \%$ da umidade de saturação, diferentemente do silicato, que tem a sua reatividade potencializada na condição de maior umidade do solo. A ação corretiva da acidez do solo por ambos os corretivos permanece crescente até 180 dias da incubação, porém, resultados satisfatórios já são obtidos com 60 dias de incubação do solo.

Palavras-chave: acidez do solo; silicato de cálcio e magnésio; calcário dolomítico, corretivos da acidez.

\section{LIME AND SILICATE APPLICATION IN SOILS INCUBATED WITH DIFFERENT MOISTURES}

\begin{abstract}
The lime is the acidity corrective more used in brazilian agricultural soils, but important benefits can be obtained from calcium and magnesium silicates. The aim of this study was to compare the effect of neutralizing soil acidity by application of lime and silicate, with incubation of the correctives in soil conditions more or less moisture. The experiment was conducted in the Soil Chemical Analysis Laboratory at the UNOESTE, using soil collected in the layer $0-20 \mathrm{~cm}$ of a Sandy Loam Argisol. The experiment was carried

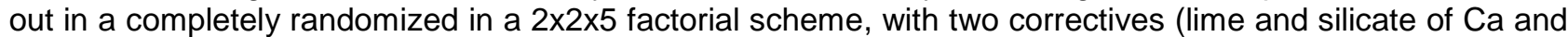
$\mathrm{Mg}$ ), two soil moistures (80 and $20 \%$ moisture soil saturation) and five times (60,90, 120, 150 and 180 days after incubation) of soil chemical properties. It was submitted to variance analysis and the effects of soil moisture and correctives were evaluated by Tukey test $(p<0,05)$ and the incubations time were evaluated by regression analysis. The silicate has a higher neutralizing capacity of soil acidity than lime, and thus its dosage should be further investigated, because the use of the calculation to determine the lime requirement can overestimate the need for silicate applied. The lime reactivity in the soil is not influenced with soil moisture between 20 and $80 \%$ moisture saturation, unlike silicate, which has its soil reactivity enhanced in the condition of higher moisture. The soil corrective action acidity by both corrective remains increasing up to 180 days of incubation, however, satisfactory results are already obtained with 60 days of soil incubation.

Keywords: soil acidity; calcium and magnesium silicate; dolomitic limestone; acidity corrective.
\end{abstract}




\section{INTRODUÇÃO}

Os solos agrícolas brasileiros, na maioria, apresentam média a alta acidez, o que traz como consequência, a baixa produtividade das culturas, em decorrência da toxidez causada por alumínio (Al) e manganês (Mn), deficiência de fósforo $(P)$ e pela baixa saturação por bases (V\%) (PADILHA et al., 2003; CAIRES et al., 2003). Assim, o cultivo agrícola dos solos ácidos exige a aplicação de corretivos, os quais ao elevarem seu $\mathrm{pH}$, neutralizam $\mathrm{O}$ efeito dos elementos tóxicos e fornecem cálcio (Ca) e magnésio $(\mathrm{Mg})$ como nutrientes, em se tratando de calcário dolomítico. Segundo Alcarde (1985), os materiais que podem ser usados na correção da acidez dos solos são aqueles que contêm como constituintes neutralizantes, óxidos, hidróxidos, carbonatos e silicatos de cálcio e ou de magnésio, tais como calcário dolomítico, calcário calcinado, óxido de cálcio ou de magnésio, hidróxido de cálcio, hidróxido de magnésio e escórias.

O calcário é o corretivo da acidez mais utilizado e, portanto, mais estudado, gerando inúmeras pesquisas sobre formas e épocas de aplicação, cálculos de doses, tempo de reação no solo, efeito residual, solubilidade, etc. No entanto, o silicato de $\mathrm{Ca}$ e $\mathrm{Mg}$ apresenta resultados satisfatórios na neutralização do Al tóxico (GALVEZ et al., 1987), na redução da acidez potencial $(\mathrm{H}+\mathrm{Al})$ e na elevação do $\mathrm{pH}$ (KORNDÖRFER et al., 1999), sendo a escória de siderurgia uma importante fonte desse silicato. De acordo com Korndörfer et al. (2002), os silicatos de $\mathrm{Ca}$ e $\mathrm{Mg}$, por apresentarem composição semelhante a dos carbonatos, podem substituir os calcários com vantagens, podendo sua recomendação de aplicação ser baseada em qualquer um dos métodos utilizados para recomendação de calagem. Segundo os autores, os benefícios proporcionados pelos silicatos de $\mathrm{Ca}$ e $\mathrm{Mg}$ estão associados ao aumento da disponibilidade de silício (Si), à elevação do $\mathrm{pH}$ e ao aumento de $\mathrm{Ca}$ e $\mathrm{Mg}$ trocáveis. Também podem reduzir a toxicidade por ferro $(\mathrm{Fe}), \mathrm{Mn}$ e $\mathrm{Al}$ às plantas e aumentar a disponibilidade de $\mathrm{P}$ no solo. No entanto, Souza et al. (2008) não recomendam a substituição da calagem pela silicatagem, pelo fato de obter piores resultados com o uso de silicato de $\mathrm{Ca}$ para os atributos químicos do solo, principalmente no que se refere os teores de $P$ remanescente, $\mathrm{Ca}, \mathrm{Al}, \mathrm{H}+\mathrm{Al}$, além de $\mathrm{pH}$ e V\%.

O uso das escórias na agricultura tem sido uma alternativa interessante para 0 aproveitamento de subprodutos das indústrias. Assim, o uso controlado desses resíduos como insumo agrícola permite o uso dessa escória para fins mais nobres, pois, além de corretivo da acidez do solo, também é fonte de $\mathrm{Si}$, considerado um elemento benéfico para as plantas (BRASIL, 2004) e que, mesmo apresentando-se em elevadas quantidades no solo (FREIRE, 2006), se encontra em baixa disponibilidade nos solos tropicais intemperizados por formar compostos de baixa solubilidade (BARBOSA FILHO et al., 2001).

Alcarde e Rodella (2003) mencionam que o silicato de cálcio é 6,78 vezes mais solúvel que o carbonato de cálcio, apresentando, portanto, maior potencial para a correção da acidez do solo em profundidade, assim como constatado por Barbosa et al. (2003), que observaram maior efeito do da silicato em relação ao calcário na elevação do $\mathrm{pH}$ até $25 \mathrm{~cm}$ de profundidade do solo. No entanto, são necessárias condições adequadas de umidade no solo para que haja solubilização em tempo razoável, visando garantir a correção do solo. De acordo com Prado e Fernandes (2001), a escória apresentou liberação mais lenta de nutrientes quando comparada ao calcário em solo cultivado com cana-de-açúcar. 
Ainda é pouco conhecida a reação dos silicatos no solo, sendo necessários estudos que determinem o poder neutralizante real desse produto, além da velocidade de reação, do efeito residual e do comportamento em condições de diferentes umidades do solo. Desta forma, objetivou-se com esse trabalho comparar o efeito neutralizante da acidez do solo por meio de calagem e silicatagem, e incubação desses corretivos em condições de solo de diferentes umidades.

\section{MATERIAL E MÉTODOS}

O experimento foi realizado no Laboratório de Análise Química de Solos da
Universidade do Oeste Paulista (Presidente Prudente-SP). O experimento teve início em novembro de $2010 \mathrm{com}$ a coleta de $50 \mathrm{dm}^{-3}$ de solo da camada de 0 a $20 \mathrm{~cm}$ de um Argissolo Vermelho-Amarelo, cuja amostra foi encaminhada para análise das propriedades químicas e granulométricas, de acordo com metodologia descrita em Raij et al. (2001) e Embrapa (1997). Os resultados das análises estão apresentados na tabela 1. Com base nos valores de $\mathrm{V} \%$ foi realizado o cálculo de necessidade de calagem e silicatagem visando elevá-lo $70 \%$.

Tabela 1. Propriedades químicas e granulométricas do solo utilizado no experimento.

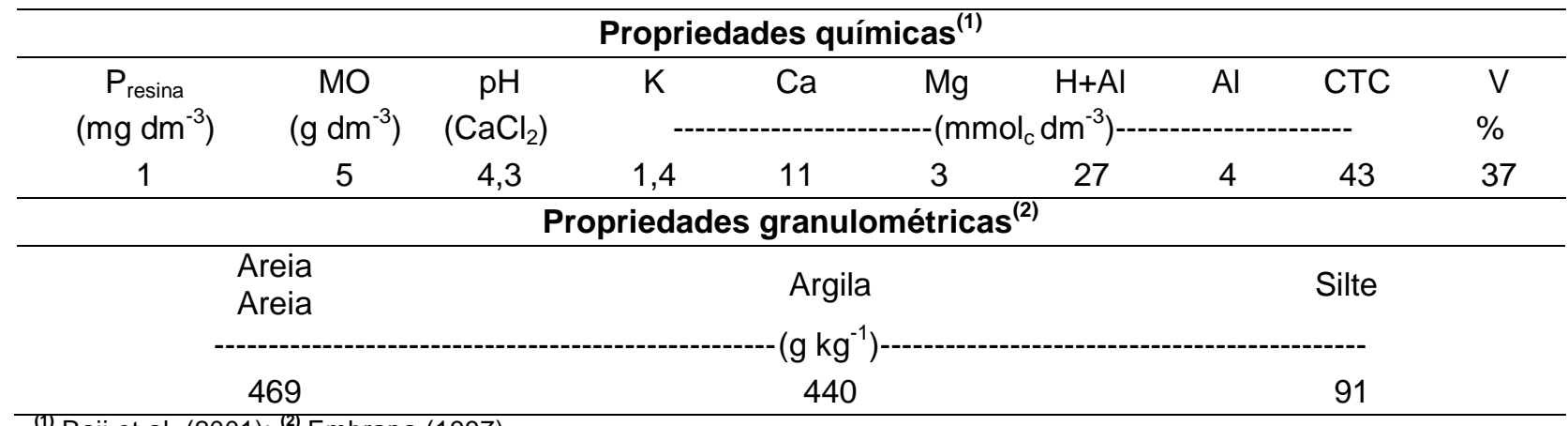

Para a correção da acidez do solo foram comparados dois corretivos, ou seja, silicato de $\mathrm{Ca}$ e $\mathrm{Mg}$ e calcário dolomítico, incubados em solos mantidos em umidades correspondentes a 20 e $80 \%$ da umidade de saturação (USat). Os efeitos dos corretivos em solos com diferentes estados de umidade foram avaliados aos 60, 90, 120, 150 e 180 dias após incubação dos mesmos. Assim, o experimento foi conduzido em delineamento inteiramente casualizado, em esquema fatorial $2 \times 2 \times 5$, com quatro repetições, constituindo 80 unidades experimentais. As unidades experimentais foram constituídas por copos plásticos de 0,20 $\mathrm{dm}^{3}$, com tampa.
Para montagem do experimento, todo o volume de solo coletado foi seco em estufa de aeração forçada a $50^{\circ} \mathrm{C}$ por 72 horas, e armazenado em sacos plásticos vedados. Em seguida, determinou-se a umidade de saturação do solo utilizado no experimento, de acordo com metodologia descrita em Embrapa (1997). Para isso, em triplicata, acomodou-se uma porção do solo seco em cilindro de aço com volume de $0,10 \mathrm{dm}^{3}$ e massa conhecida. A massa do conjunto cilindro-solo seco foi determinada, e em seguida, a amostra foi colocada em bandeja contendo água até $2 / 3$ da altura do cilindro, por 24 horas, para saturação da amostra com água. Para evitar a perda de solo, foi colocado um tecido de poliéster na 
face inferior do cilindro com o auxílio de um atilho de borracha. Ao final do período de saturação, retirou-se o excesso de água da amostra com papel absorvente e determinou-se a massa da amostra saturada. Com a diferença de massa inicial e final, calculou-se a Usat, cujo resultado foi de $0,40 \mathrm{~kg} \mathrm{~kg}^{-1}$. Conhecendo-se a Usat, calculou-se o volume de água necessário para umedecer $0,20 \mathrm{dm}^{3}$ de solo seco (volume de cada unidade experimental), com densidade aproximada de $1,0 \mathrm{~kg} \mathrm{dm}^{-3}$, para que o mesmo atingisse 20 e $80 \%$ da Usat.

As doses dos corretivos, calculadas objetivando elevar a $\mathrm{V} \%$ a $70 \%$, consideraram os valores de PRNT informadas pelos fornecedores, ou seja, PRNT de $90 \%$ e $80 \%$ para o calcário dolomítico e para o silicato de $\mathrm{Ca}$ e $\mathrm{Mg}$, respectivamente.

Em dezembro de 2010, após a aplicação dos tratamentos de umidade e corretivos, os solos foram acondicionados em recipientes plásticos com tampa, com capacidade de 0,20 $\mathrm{dm}^{-3}$ mantidos sobre bancadas em local com temperatura variando de 25 a $30^{\circ} \mathrm{C}$. A cada sete dias os recipientes foram abertos por 5 a
10 segundos para permitir as trocas gasosas resultantes da reação dos corretivos no solo. Em cada época de avaliação encaminhou-se o solo de 16 unidades experimentais para avaliação dos parâmetros: $\mathrm{pH}, \mathrm{H}+\mathrm{Al}, \mathrm{Al}, \mathrm{Ca}$, $\mathrm{Mg}$, potássio $(\mathrm{K})$, $\mathrm{P}$, capacidade de troca catiônica (CTC) e V\%.

Os resultados foram comparados por meio de análise de variância (Teste $\mathrm{F}$ a $5 \%$ de probabilidade) e as variáveis umidades do solo e corretivos foram submetidas ao teste de Tukey $(p<0,05)$. Já o efeito da época de amostragem foi analisado por meio de análise de regressão, sendo escolhidos modelos matemáticos com ajustes significativos a $1 \mathrm{e}$ $5 \%$ de probabilidade pelo teste $\mathrm{F}$.

\section{RESULTADOS E DISCUSSÂO}

Por meio da análise de variância dos resultados (Tabela 2 ) verificou-se que 0 corretivo da acidez utilizado, a umidade do solo e o tempo de reação influenciaram na acidez e nos parâmetros da fertilidade do solo, e dependendo do parâmetro avaliado, houve interação significativa entre esses fatores.

Tabela 2. Valores de $F$ calculados pela análise de variância para os resultados de acidez ativa $(\mathrm{pH})$, acidez potencial $(\mathrm{H}+\mathrm{Al})$, fósforo $(\mathrm{P})$, potássio $(\mathrm{K})$, cálcio $(\mathrm{Ca})$, magnésio $(\mathrm{Mg})$, capacidade de troca catiônica $(\mathrm{CTC})$ e saturação por bases (V\%), em função dos corretivos da acidez do solo (calcário e silicato), aplicados em diferentes umidades do solo (20 e 80 \% da umidade de saturação) e do tempo de incubação (60, 90, 120, 150 e 180 dias).

\begin{tabular}{|c|c|c|c|c|c|c|c|c|}
\hline $\begin{array}{l}\text { Causas de } \\
\text { Variação }\end{array}$ & $\mathrm{pH}$ & $\mathrm{H}+\mathrm{Al}$ & $\mathbf{P}$ & $\mathbf{K}$ & $\mathrm{Ca}$ & $\mathrm{Mg}$ & СтC & V\% \\
\hline Corretivo (C) & $885.94^{* *}$ & $911.53^{* *}$ & $1.53 \mathrm{~ns}$ & $2.67 n s$ & $285.15^{\star *}$ & $0.67 n s$ & $96.79^{\star \star}$ & $724.25^{\star *}$ \\
\hline Umidade $(U)$ & $221.48^{\star *}$ & $85.28^{\star \star}$ & $0.90 \mathrm{~ns}$ & $1.02 \mathrm{~ns}$ & $28.86^{\star *}$ & $0.24 \mathrm{~ns}$ & $9.83^{* *}$ & $55.90^{* *}$ \\
\hline Tempo (T) & $39.41^{* *}$ & $87.79^{* *}$ & $1.84 \mathrm{~ns}$ & $50.65^{* *}$ & $105.92^{* *}$ & $32.70^{\star *}$ & $72.49^{* *}$ & $212.57^{\star *}$ \\
\hline $\mathrm{CxU}$ & $2.964^{* *}$ & $191.89^{* *}$ & $1.81 \mathrm{~ns}$ & $1.66 \mathrm{~ns}$ & $19.51^{* *}$ & $0.03 \mathrm{~ns}$ & $3.82 \mathrm{~ns}$ & $78.69^{* *}$ \\
\hline CxT & $0.01 \mathrm{~ns}$ & $1.02 \mathrm{~ns}$ & $0.88 \mathrm{~ns}$ & $1.70 \mathrm{~ns}$ & $4.03^{\star *}$ & $1.21 \mathrm{~ns}$ & $2.49 \mathrm{~ns}$ & $7.12^{\star *}$ \\
\hline UxT & $1.89 \mathrm{~ns}$ & $1.78 \mathrm{~ns}$ & $0.97 \mathrm{~ns}$ & $1.19 \mathrm{~ns}$ & $0.52 \mathrm{~ns}$ & $1.18 \mathrm{~ns}$ & $0.64 n s$ & $0.66 n s$ \\
\hline$C V(\%)$ & 1.55 & 4.36 & 42.84 & 10.74 & 14.73 & 12.15 & 10.40 & 2.20 \\
\hline
\end{tabular}


A utilização de silicato de $\mathrm{Ca}$ e $\mathrm{Mg}$ resultou em maior correção da acidez do solo em relação ao calcário dolomítico, com maiores valores do $\mathrm{pH}$ e menores de $\mathrm{H}+\mathrm{Al}$, independente da umidade do solo (Tabela 3 ). Ramos et al. (2006) também observaram maior eficiência de silicatos na elevação do $\mathrm{pH}$ do solo em relação ao calcário. Já Veloso et al. (1992) relatam maior efeito do calcário dolomítico na correção da acidez do solo em relação à escória, com 75 dias de incubação no solo. No entanto, esses autores compararam o efeito de doses de calcários e silicatos sem considerar o poder neutralizante e a reatividade dos produtos testados. Louzada (1987) indicaram que as escórias são um pouco menos eficientes na elevação do $\mathrm{pH}$ do solo que o calcário, sendo essas diferenças atribuídas ao valor neutralizante mais baixo da escória (86\%) comparado ao do calcário (100\%). Nesse sentido, é importante salientar que no presente estudo as doses dos corretivos não foram as mesmas, pois considerou-se o PRNT dos materiais e as doses foram calculadas de acordo a metodologia da saturação por bases descrita em Raij et al. (1996).

Tabela 3. Desdobramento das médias de acidez ativa $(\mathrm{pH})$, acidez potencial $(\mathrm{H}+\mathrm{Al})$, fósforo $(\mathrm{P})$, potássio $(\mathrm{K})$, cálcio $(\mathrm{Ca})$ e saturação por bases $(\mathrm{V} \%)$ em função da interação entre as fontes de corretivos da acidez do solo (calcário e silicato) e umidade do solo ( $20 \%$ ou $80 \%$ da umidade de saturação).

\begin{tabular}{|c|c|c|c|}
\hline \multirow{2}{*}{ Corretivo } & \multicolumn{2}{|c|}{ Umidade do solo } & \multirow[b]{2}{*}{ Média } \\
\hline & $20 \%$ & $80 \%$ & \\
\hline \multicolumn{4}{|c|}{ pH } \\
\hline Calcário & $6,2 \mathrm{bA}$ & $6,2 \mathrm{bA}$ & 6,2 \\
\hline Silicato & $6,5 \mathrm{aB}$ & $7,3 \mathrm{aA}$ & 6,9 \\
\hline Média & 6,4 & 6,7 & \\
\hline \multicolumn{4}{|c|}{$\mathrm{H}+\mathrm{Al}\left(\mathrm{mmol}_{\mathrm{c}} \mathrm{dm}^{-3}\right)$} \\
\hline Calcário & 16 bA & $17 \mathrm{bA}$ & 17 \\
\hline Silicato & $14 \mathrm{aB}$ & $11 \mathrm{aA}$ & 12 \\
\hline Média & 15 & 14 & \\
\hline \multicolumn{4}{|c|}{$P\left(\mathrm{mg} \mathrm{dm}^{-3}\right)$} \\
\hline Calcário & 2 & 2 & $2 \mathrm{a}$ \\
\hline Silicato & 2 & 3 & $3 a$ \\
\hline Média & $2 \mathrm{~A}$ & $2 \mathrm{~A}$ & \\
\hline \multicolumn{4}{|c|}{$\mathrm{K}\left(\mathrm{mmol}_{\mathrm{c}} \mathrm{dm}^{-3}\right)$} \\
\hline Calcário & 1,2 & 1,2 & $1,2 \mathrm{a}$ \\
\hline Silicato & 1,4 & 1,2 & $1,3 \mathrm{a}$ \\
\hline Média & $1,3 \mathrm{~A}$ & $1,2 \mathrm{~A}$ & \\
\hline \multicolumn{4}{|c|}{$\mathrm{Ca}\left(\mathrm{mmol}_{\mathrm{c}} \mathrm{dm}^{-3}\right)$} \\
\hline Calcário & $25 \mathrm{bA}$ & 26 aA & 26 \\
\hline Silicato & $40 \mathrm{aA}$ & $51 \mathrm{bB}$ & 46 \\
\hline Média & 33 & 39 & \\
\hline \multicolumn{4}{|c|}{$\mathrm{Mg}\left(\mathrm{mmol}_{\mathrm{c}} \mathrm{dm}^{-3}\right)$} \\
\hline Calcário & 20 & 20 & $20 \mathrm{a}$ \\
\hline Silicato & 20 & 20 & $20 \mathrm{a}$ \\
\hline Média & $20 \mathrm{~A}$ & $20 \mathrm{~A}$ & \\
\hline \multicolumn{4}{|c|}{ CTC $\left(\mathrm{mmol}_{\mathrm{c}} \mathrm{dm}^{-3}\right)$} \\
\hline Calcário & 62 & 64 & $63 \mathrm{~b}$ \\
\hline Silicato & 75 & 84 & $80 \mathrm{a}$ \\
\hline Média & $69 \mathrm{~B}$ & $74 \mathrm{~A}$ & \\
\hline \multicolumn{4}{|c|}{ V\% } \\
\hline Calcário & $73 \mathrm{bA}$ & $73 \mathrm{bA}$ & 73 \\
\hline Silicato & $80 \mathrm{aB}$ & $86 \mathrm{aA}$ & 83 \\
\hline Média & 77 & 79 & \\
\hline
\end{tabular}


Castro (2009), ao avaliar o efeito da correção da acidez do solo aos 180 dias após a aplicação superficial de 4,0 e 4,5 $\mathrm{t} \mathrm{ha}^{-1}$ de calcário dolomítico e silicato de $\mathrm{Ca}$ e $\mathrm{Mg}$, respectivamente em Sistema Semeadura Direta (dose calculada visando elevar $\mathrm{V} \%$ a 70 ), verificou que ambos os corretivos elevaram o $\mathrm{pH}$ de forma semelhante na camada mais superficial $(0$ a $5 \mathrm{~cm}$ ). Porém, na camada de 5 a $20 \mathrm{~cm}$, somente o silicato apresentou efeito na elevação do $\mathrm{pH}$. Corrêa et al. (2007) obtiveram resultados semelhantes, porém com efeito do silicato até $40 \mathrm{~cm}$ de profundidade após 90 dias da aplicação. Esses resultados podem ser explicados devido à maior solubilidade do silicato, que segundo Alcarde e Rodella (2003), é 6,78 vezes mais solúvel que o carbonato de $\mathrm{Ca}$. A maior solubilidade do silicato em relação ao calcário ficou evidente nos resultados apresentados na Tabela 3 , em que não se observa influência da umidade do solo na ação do calcário, diferentemente do silicato, em que a condição de maior umidade potencializa o efeito do corretivo na redução da acidez do solo.

$O$ efeito dos corretivos da acidez do solo também pode ser observado nos resultados de CTC e V\%, sendo a silicatagem mais eficiente em aumentar esses parâmetros, principalmente em condições de maior umidade do solo (Tabela 3). Assim, a utilização de silicato promoveu melhoria na fertilidade do solo em relação ao calcário, já que, por se tratar de um solo tropical, portanto, de carga variável e dependente do $\mathrm{pH}$, aumentou a CTC do solo, além de aumentar a ocupação do complexo de troca por cátions básicos, resultando em maior $\mathrm{V} \%$. O aumento da $\mathrm{V} \%$ pelo uso de silicato, principalmente na umidade referente a $80 \%$ da USat, deve-se à redução da acidez potencial $(\mathrm{H}+\mathrm{Al})$ e ao aumento dos teores de Ca trocável nessas condições, já que os tratamentos aplicados não influenciaram nos teores de $\mathrm{K}$ e $\mathrm{Mg}$ disponíveis (Tabela 3). Os valores de $K$ no solo não sofreram alterações em relação à análise inicial, realizada antes da aplicação dos corretivos (Tabela 1), pois os corretivos utilizados não são fontes de $\mathrm{K}$, e seus efeitos pouco interferem na disponibilidade de $\mathrm{K}$ no solo, conforme relatado por Caires et al. (2006), Caires et al. (2000), Castro (2009) e Alleoni et al. (2005).

Diferentemente dos resultados obtidos para $\mathrm{K}$, os teores de $\mathrm{Ca}$ e $\mathrm{Mg}$ no solo atingiram valores muito acima dos encontrados inicialmente no solo, apesar do teor de Mg não sofrer influência significativa dos tratamentos (Tabela 3). Ramos et al. (2006), ao comparar o efeito do silicato de $\mathrm{Ca}$ e $\mathrm{Mg}$ com o calcário calcítico, ambos incubados ao solo por 40 dias, verificaram que esses insumos apresentaram capacidades semelhantes em aumentar o teor de Ca trocável no solo, e maior capacidade do silicato em disponibilizar Mg. Castro (2009), em aplicação superficial de calcário dolomítico e silicato de $\mathrm{Ca}$ e $\mathrm{Mg}$, em solo sob Sistema Semeadura Direta, verificou que, transcorridos 180 dias da aplicação, tanto o calcário como o silicato aumentaram os teores de $\mathrm{Ca}$ (na camada de 0 a $20 \mathrm{~cm}$ ) e $\mathrm{Mg}$ (na camada de 0 a $10 \mathrm{~cm}$ ) em relação tratamento testemunha, não havendo diferença significativa entre os corretivos.

$O$ teor de $P$ no solo não sofreu influência da correção da acidez promovida por ambos os corretivos (Tabela 3), seja com incubação em solo com maior ou menor umidade, apesar de haver na literatura resultados consistentes de aumento da disponibilidade de $\mathrm{P}$ com a aplicação de corretivos da acidez (NOVAIS et al., 2007). Como o ânion silicato é quimicamente adsorvido ao solo, há, a princípio, uma competição entre o $\mathrm{Si}$ e o $\mathrm{P}$ pelos mesmos sítios de adsorção, deslocando $P$ adsorvido para a solução 
(OBIHARA et al., 1972). Smyth e Sanches (1980) verificaram redução na adsorção de $P$ na ordem de 18 e $24 \%$ para solos (inicialmente com $\mathrm{pH}$ de 4,8) que receberam calagem e silicatagem, respectivamente. Carvalho (2000) relataram que a ação do silicato na dessorção de $P$ é mais evidente em solo menos intemperizados, com maior teor de caulinita, do que no solo mais intemperizado, com maior teor de gibbsita.

O solo utilizado para a incubação dos corretivos teve seu teor de $\mathrm{P}$ disponível aumentado de $1,0 \mathrm{mg} \mathrm{dm}^{-3}$ (Tabela 1) para 2,3 $\mathrm{mg} \mathrm{dm}^{-3}$, mesmo em condições de $\mathrm{pH}$ acima de 6,0 , indicando haver reduzida quantidade $P$ não-lábil no solo, ou seja, adsorvidos a oxihidróxidos de Fe e Al (NOVAIS et al., 2007). Castro (2009) observou elevações expressivas nos teores de $\mathrm{P}$ no solo, tanto com a realização de silicatagem como de calagem, aumentando o teor de $\mathrm{P}$ na camada 0 a $5 \mathrm{~cm}$ em relação à testemunha. Por outro lado, a aplicação de silicato proporcionou maiores valores de $\mathrm{P}$ na camada de 5 a $10 \mathrm{~cm}$ em comparação à testemunha (sem utilização de corretivo) e aos tratamentos que receberam aplicação de calcário, indicando ser um corretivo de maior solubilidade. Putz et al. (2008), estudando a aplicação de silicato e calcário em solos de baixa fertilidade e teor de argila de $330 \mathrm{~g} \mathrm{~kg}^{-1}$, também verificaram maior teor de $\mathrm{P}$ disponível em solo corrigido com silicato de $\mathrm{Ca}$ e $\mathrm{Mg}$ em relação ao calcário, apesar de não observarem diferenças em relação a $\mathrm{pH}, \mathrm{H}+\mathrm{Al}, \mathrm{K}, \mathrm{Ca}, \mathrm{Mg}$ e V\%, acarretando em maiores teores foliares de $\mathrm{N}$ e $\mathrm{P}$ na cultura da batata. Como no presente experimento, Putz et al. (2008) também não constataram efeito da umidade do solo na ação dos corretivos da acidez nos atributos químicos do solo.

O tempo de incubação para a reação dos corretivos também influenciou na correção da acidez e na fertilidade do solo. A calagem e a silicatagem aumentaram o $\mathrm{pH}\left(\mathrm{CaCl}_{2}\right)$ (Figura $1 \mathrm{~A}$ ) e reduziram o $\mathrm{H}+\mathrm{Al}$ (Figura $1 \mathrm{~B}$ ) de forma linear entre 60 e 180 dias após a incubação. No entanto, é importante ressaltar que, já aos 60 dias após a incubação, o pH que inicialmente era de 4,3 aumentou em média para 6,1 e 6,8 com a aplicação de calcário e silicato de $\mathrm{Ca}$ e $\mathrm{Mg}$ respectivamente. Aos 180 dias de incubação os valores de pH subiram para $6,4 \mathrm{e}$ 7,0 , para os tratamentos com calagem $\mathrm{e}$ silicatagem, respectivamente (Figura 1A). Nesse caso, observa-se que, no caso da calagem, o pH do solo permaneceu dentro da faixa ideal para a máxima disponibilidade de nutrientes para as plantas, nesse período de avaliação, ou seja, entre 60 e 180 dias após incubação. Já o tratamento com silicato de $\mathrm{Ca}$ e $\mathrm{Mg}$ proporcionou valores de $\mathrm{pH}$ muito elevados, que podem comprometer a disponibilidade de nutrientes, principalmente, de micronutrientes catiônicos (MALAVOLTA, 2006). 


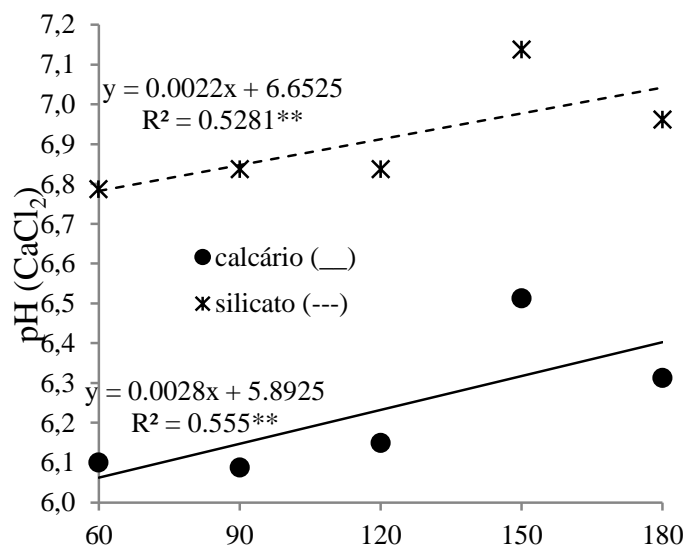

(A)

Tempo de incubação (dias)

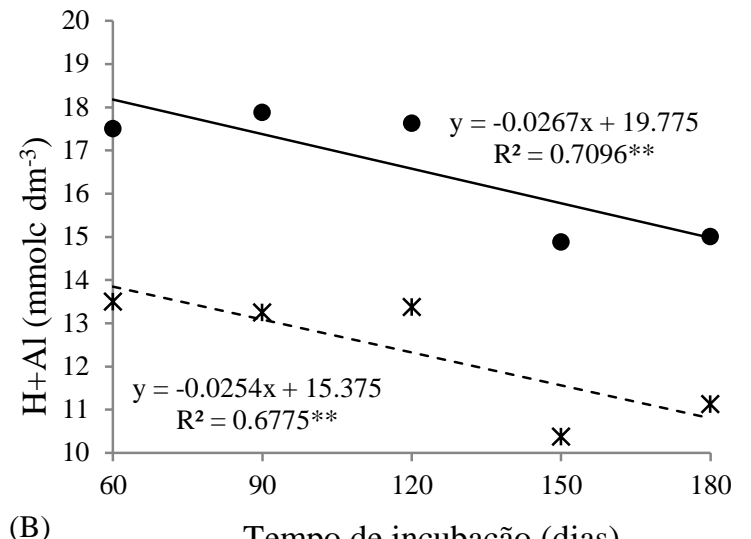

Tempo de incubação (dias)

Figura 1. Resultados de $\mathrm{pH}(\mathrm{A})$ e $\mathrm{H}+\mathrm{Al}(\mathrm{B})$ em função do corretivo da acidez utilizado (calcário ou silicato) e do tempo de reação dos corretivos no solo $\left(60,90,120,150\right.$ e 180 dias). ${ }^{*}$ Significativo a $1 \%$ de probabilidade pelo teste $\mathrm{F}$.

A V\% (Figura 2A) e a CTC (Figura 2B) aumentaram de forma quadrática em função do tempo de incubação tanto do calcário como do silicato. Esse resultado na V\% foi influenciado pelos aumentos também quadráticos dos teores de Ca (Figura 3B) e (Figura 3C) Mg disponíveis para ambos os corretivos, já que o teor de $\mathrm{K}$ trocável (Figura 3A) não sofreu influência dos tratamentos, e pela redução linear dos teores de $\mathrm{H}+\mathrm{Al}$. A V\%, que inicialmente era de 37 , e que foi o parâmetro utilizado como referência para cálculo das doses de corretivos, alcançou valor muito próximo ao desejado (70\%) já aos 60 dias após a incubação, quando o corretivo utilizado foi o calcário. Nessa mesma época de avaliação (primeira época) a incubação do solo com silicato de $\mathrm{Ca}$ e $\mathrm{Mg}$ resultou em V\% próximo a 80 , evidenciando a maior eficiência do silicato em relação ao calcário. Dessa forma, o uso da metodologia de cálculo de necessidade de calagem (RAlJ et al., 1996) para recomendar a necessidade de silicatagem pode superestimar a dose de silicato.

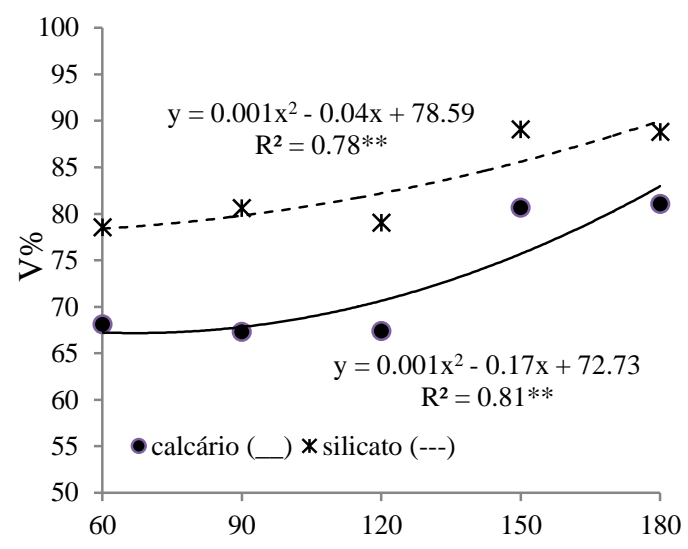

(A)

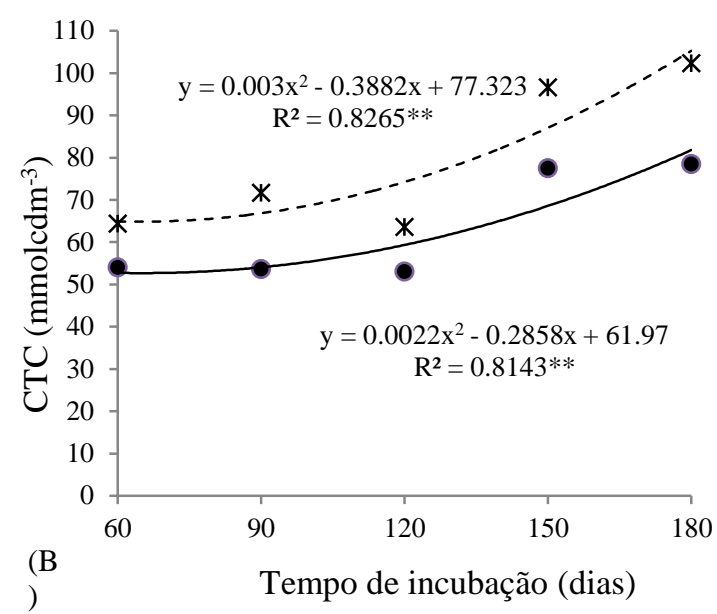

Figura 2. Resultados de V\% (A) e CTC (B), em $\mathrm{mmol}_{\mathrm{c}} \mathrm{dm}^{-3}$, em função do corretivo da acidez utilizado (calcário e silicato) e do tempo de reação dos corretivos no solo (60, 90, 120, 150 e 180 dias). ${ }^{*}$ Significativo a $1 \%$ de probabilidade pelo teste $\mathrm{F}$. 

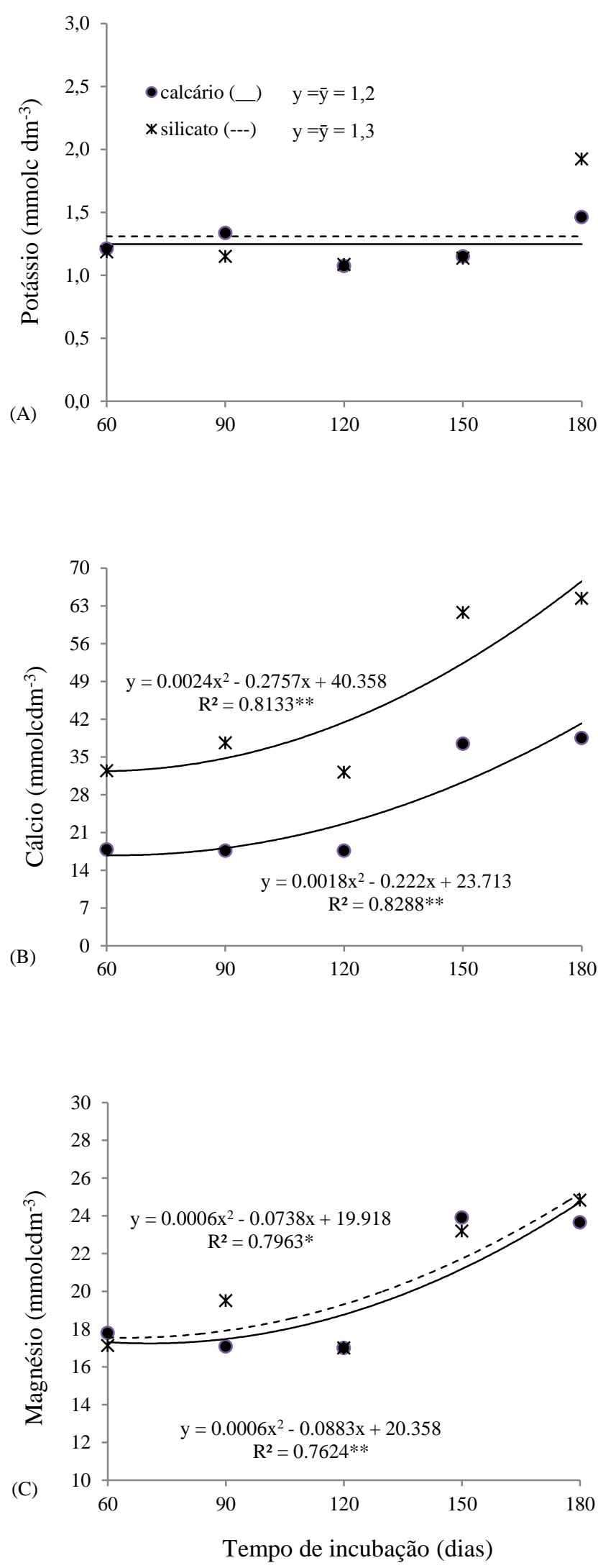

Figura 3. Teores de potássio $(A)$, cálcio $(B)$ e magnésio $(C)$, em $\mathrm{mmol}_{\mathrm{c}} \mathrm{dm}^{-3}$, em função do corretivo da acidez utilizado (calcário e silicato) e do tempo de reação dos corretivos no solo (60, 90, 120, 150 e 180 dias). ${ }^{*}$,* Significativo a 5 e $1 \%$ de probabilidade pelo teste $\mathrm{F}$, respectivamente. 


\section{CONCLUSÕES}

$\mathrm{O}$ silicato de $\mathrm{Ca}$ e $\mathrm{Mg}$ apresenta maior de capacidade neutralizante da acidez do solo que o calcário dolomítico, e com isso sua dosagem deve ser melhor estudada, pois a utilização do cálculo usado para determinar a necessidade de calagem pode superestimar a necessidade de silicatagem.

A reatividade do calcário no solo não é influenciada quando o solo está com umidade entre 20 e $80 \%$ da umidade de saturação, diferentemente do silicato, que tem a sua reatividade potencializada na condição de maior umidade do solo.

A ação corretiva da acidez do solo promovida tanto pelo calcário como pelo silicato de $\mathrm{Ca}$ e $\mathrm{Mg}$ permanece crescente até 180 dias da incubação, porém, resultados satisfatórios já são obtidos com 60 dias de incubação do solo.

\section{REFERÊNCIAS}

ALCARDE, J.C. Corretivos da acidez dos solos: características de qualidade. In: MALAVOLTA, E. (Ed.) Seminário sobre corretivos agrícolas. Piracicaba: Fundação Cargil, 1985. p. 97-117.

ALCARDE, J.A.; RODELLA, A.A. Qualidade e legislação de fertilizantes e corretivos. In: CURI, N. et al. (Eds.) Tópicos em Ciência do Solo. Viçosa: Sociedade Brasileira de Ciência do Solo, 2003. p. 291-334.

ALLEONI, L.R.F.; CAMBRI, M.A.; CAIRES, E.F.; Atributos químicos de um Latossolo de Cerrado sob plantio direto de acordo com doses e formas de aplicação de calcário. Revista Brasileira de Ciência do Solo, v. 29, n. 6, p. 923-934, 2005. http://dx.doi.org/10.1590/S0100-

06832005000600010

BARBOSA, D.S.; CAMARGO, M.S.; RAMOS, L.A.; RESENDE, R.H.; ARRUDA, D.; KORNDÖRFER, G.H.; PEREIRA, H.S. Comparação entre silicato e calcário na correção do solo e no fornecimento de silício em profundidade. In: 29o Congresso Brasileiro de Ciência do Solo, Ribeirão Preto. Anais... Sociedade Brasileira de Ciência do Solo, 2003. CD-ROM.

BARBOSA FILHO, M.P.; SNYDER, G.H.; FAGERIA, N.K.; DATNOFF, L.E. ; SILVA, O.F.
Silicato de cálcio como fonte de silício para o arroz de sequeiro. Revista Brasileira de Ciência do Solo, v. 25, n. 2, p. 325-30, 2001.

BRASIL. Decreto-lei no 2.954, de 14 de janeiro de 2004. Aprova o regulamento da lei no 6894 de 16 de janeiro de 1980, que dispõe sobre a inspeção e fiscalização da produção e do comércio de fertilizantes, corretivos, inoculantes ou biofertilizantes destinados à agricultura e da outras providências. Normas jurídicas. Brasília: 2004. 27 p.

CAIRES, E.F.; BANZATTO, D.A; FONSECA, A.F. Calagem na superfície em sistema plantio direto. Revista Brasileira de Ciência do Solo, v. 24, n. 1, p. 161-169, 2000.

CAIRES, E.F. et al. Crescimento radicular e nutrição do milho em resposta ao calcário e gesso aplicados na implantação do sistema plantio direto. In: 29ㅇ Congresso Brasileiro de Ciência do Solo, Ribeirão Preto. Anais... Sociedade Brasileira de Ciência do Solo, 2003. CD-ROM.

CAIRES, E.F.; KUSMAN, M.T.; BARTH, G.; GARBUIO, F.J.; PADILHA, J.M. Aplicação de corretivos superficial e cobertura de aveia preta antecedendo os cultivos de milho e soja em sistema plantio direto. Revista Brasileira de Ciência do Solo, v. 30, n. 1, p. 87-98, 2006. http://dx.doi.org/10.1590/S010006832006000100010

CARVALHO, R. Dessorção de fósforo por silício em solos cultivados com eucalipto. Revista Brasileira de Ciência do Solo, v. 24, n. 1, p. 6974, 2000

CASTRO, G.S.A. Alterações físicas e químicas do solo em função do sistema de produção e da aplicação superficial de silicato e calcário. 2009. 135 p. Dissertação (Mestrado) Universidade Estadual Paulista, Botucatu.

CORRÊA, J.C.; BÜLL, L.T.; CRUSCIOL, C.A.C.; MARCELINO, R. Correção da acidez e mobilidade de íons em Latossolo com aplicação superficial de escória, lama cal, lodos de esgoto e calcário. Pesquisa Agropecuária Brasileira, v.42, n.9, p.1307-1317, 2007. http://dx.doi.org/10.1590/S0100-

204X2007000900013

EMBRAPA. Serviço Nacional de Pesquisa do Solo. Manual de métodos de análises de solo. Rio de Janeiro: Embrapa Solos, 1997. 212 p.

FREIRE, O. Solos das regiões tropicais.

Botucatu: FEPAF, 2006. 268 p. 
GALVEZ, L.; CLARK, R.B.; GOURLEY, L.M.; MARANVILLE, J.W. Silicon interactions with manganese and aluminum toxicity in sorghum. Journal of Plant Nutrition, v. 10, n. 9-16, p. 1139-1147, 1987.

KORNDÖRFER, G.H.; ARANTES, V.A.; CORREAA, G.F.; SNYDER, G.H. Efeito do silicato de cálcio no teor de silício no solo e na produção de grãos de arroz de sequeiro. Revista Brasileira de Ciência do Solo, v. 23, n. 3, p. 635-641,1999.

KORNDÖRFER, G.H.; PEREIRA, H.S.; CAMARGO, M.S. Silicatos de cálcio e magnésio na agricultura. Uberlândia: GPSiICIAG-UFU, 2002. 23 p. (Boletim Técnico, 1)

LOUZADA, P.T.C. Eficiência de uma escória de siderurgia como corretivo e fertilizante do solo. 1987. 52 f. Dissertação (Mestrado) Universidade Federal de Viçosa, Viçosa.

MALAVOLTA, E. Manual de nutrição mineral de plantas. São Paulo: Agronômica Ceres, 2006.

NOVAIS, R.F.; SMYTH, T.J.; NUNES, F.N. FÓSFORO. In: NOVAIS, R.F. et al. (Eds.) Fertilidade do solo. Viçosa: Sociedade Brasileira de Ciência do Solo, 2007.

OBIHARA, C.H.; RUSSEL, E.W. Specific adsorption of the silicate and phosphate by soils. Journal of Soil Science, v. 23, n. 1, p. 105-117, 1972. http://dx.doi.org/10.1111/j.13652389.1972.tb01646.x

PADILHA, J.M.; BARTH, G.; GARBUIO, F.J.; KUSMAN, M.T.; CAIRES, E.F. Aplicação superficial de calcário calcítico e dolomítico em sistema plantio direto. In: 29ㅇ Congresso Brasileiro de Ciência do Solo, Ribeirão Preto. Anais... Sociedade Brasileira de Ciência do Solo, 2003. CD-ROM.

PRADO, R.M.; FERNANDES, F.M. Resposta da cana-de-açúcar à aplicação da escória de siderurgia como corretivo de acidez do solo. Revista Brasileira de Ciência do Solo, v. 25, n. 1, p. 201-209, 2001.

PUTZ, A.L.; CRUSCIOL, C.A.C.; LEMOS, L.B.; SORATTO, R.P. Influência de silicato e calcário na nutrição, produtividade e qualidade de batata sob deficiência hídrica. Revista Brasileira de Ciência do Solo, v. 32, n. 4, p. 1651-1659, 2008. http://dx.doi.org/10.1590/S0100$\underline{06832008000400030}$

RAIJ, B. van; CANTARELLA, H.; QUAGGIO, J.A.; FURLANI, A.M.C. Recomendações de adubação e calagem para o Estado de São Paulo. 2. ed. Campinas: Instituto Agronômico de Campinas, 1996.
RAIJ, B. van, ANDRADE, J.C.; CANTARELLA, H.; QUAGGIO, J.A. Análise química para avaliação da fertilidade de solos tropicais. Campinas, Instituto Agronômico, 2001.

RAMOS, L.A.; NOLLA, A.; KORNDÖRFER, G.H.; PEREIRA, H.S.; CAMARGO, M.S. DE Reatividade de corretivos da acidez e condicionadores de solo em colunas de lixiviação. Revista Brasileira de Ciência do Solo, v. 30 , n.5, p.849-857, 2006. http://dx.doi.org/10.1590/S0100$\underline{06832006000500011}$

SMYTH, T.J.; SANCHEZ, P.A. Effects of lime, silicate, and phosphorus applications to na Oxisol on phosphorus sorption and íon retention. Soil Science Society of American Journal, v. 44, n. 3, p. 500-505, 1980. http://dx.doi.org/10.2136/sssaj1980.03615995004 400030012x

SOUZA, R.F.; FAQUIN, V.; CARVALHO R.; TORRES, P.R.F.; POZZA, A.A.A. Atributos químicos de solos influenciados pela substituição do carbonato por silicato de cálcio. Revista Brasileira de Ciência do Solo, v. 32, n. 4, p. 1563-1572, 2008. http://dx.doi.org/10.1590/S010006832008000400020

VELOSO, C.A.C.; BORGES, A.L.; MUNIZ, A.S.; VEIGAS, I.A. DE J.M. Efeito de diferentes materiais no pH do solo. Scientia Agricola, v. 49, n. 1, p. 123-128, 1992. 